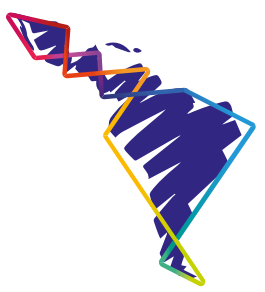

\title{
Pedagogía teatral y Derechos Humanos: una puerta para el autoconocimiento
}

\section{Theater pedagogy and Human Rights: a door to self-knowledge}

\section{Teatro pedagogia e Direitos Humanos: uma porta para o autoconhecimento}

\author{
Sandra S. Burmeister G. ${ }^{1}$
}

\begin{abstract}
Resumen
La importancia de la investigación educativa en derechos humanos a través de la pedagogía teatral es que aborda una metodología activa en el aula, la cual permite comprender contenidos teóricos mediante la vivencia individual y grupal, desde una perspectiva cotidiana de autoconocimiento. Esto significa que sensibiliza a los participantes en derechos humanos, aproximándose al conflicto con el juego teatral, que estimula el pensamiento divergente, y el diálogo controversial, que desarrolla la reflexión crítica orientada a un nuevo conocimiento. El método de investigación fue cualitativo, ya que se trabajó desde un enfoque de derecho y en torno a los aspectos cualitativos del sujeto de derecho. Lo anterior, con base en la observación participante y no participante, un grupo focal, entrevistas y análisis de contenidos. En este caso, el universo escogido fue un grupo acotado de profesores y profesoras de educación escolar. Para registrar la información obtenida, se utilizaron instrumentos tales como formularios, entrevistas, una
\end{abstract}

Recibido: 24-1-2021 - Aceptado: 13-5-2021

1 Magíster en Educación en Derechos Humanos - Centro de Cooperación Regional para la Educación de Adultos en América Latina y El Caribe (CREFAL) - . Pedagoga teatral (Pontificia Universidad Católica de Chile). Diplomada en Literatura para la Infancia, Adolescencia y Juventud (Universidad de Chile). Relacionadora pública (Universidad del Pacífico). Actriz (Escuela de Comunicación Escénica de Patricio Achurra y Eduardo Mujica). Integradora de saberes, escritora y charlista. Investigadora de educación en derechos humanos a través de la pedagogía teatral, para el pleno desarrollo de las personas, como agente inspirador de la identidad y transformación social. Actualmente, forma parte del equipo docente del Diplomado de Pedagogía Teatral en la Pontificia Universidad Católica de Chile. Es integrante activa de la Red de Equipos de Educación en Derechos Humanos. De nacionalidad chilena. Correo electrónico: ssburgar@gmail.com (D) https://orcid.org/0000-0002-8316-8890 
charla y una práctica docente con el grupo etario. En conclusión, esta fue una investigación interactiva y participativa que propició a la didáctica y arrojó datos relevantes para la sistematización educativa en derechos humanos a través de la pedagogía teatral.

Palabras clave: juego teatral, derechos humanos, conflicto y diálogo.

\begin{abstract}
The importance of research on human rights education through theater pedagogy is that it addresses an active methodology in the classroom that allows understanding theoretical content through individual and group experience from a daily perspective of self-knowledge. This signifies that it sensitizes the participants in human rights, addressing the conflict through theatrical play, which stimulates divergent thinking, and the controversial dialogue that develops critical thinking leading to new knowledge. The research method was qualitative, since it was worked from a legal approach and around the qualitative aspects of the subject of law. Execution was based on participant and non-participant observation, the creation of a focus group, interviews and content analysis. In this case, the chosen universe was a limited group of school education teachers. Instruments such as forms, interviews, talks and teaching practice with the age group were used to record the information obtained. In conclusion, it was an interactive and participatory investigation that fostered didactics and yielded relevant data for the systematization of human rights education through theater pedagogy.
\end{abstract}

Keywords: Theatrical play; Human rights; Conflict and dialogue.

\title{
Resumo
}

A importância da pesquisa sobre educação em direitos humanos por meio da pedagogia do teatro é que ela aborda uma metodologia ativa na sala de aula que permite a compreensão do conteúdo teórico por meio da vivência individual e grupal em uma perspectiva cotidiana de autoconhecimento. Isso significa que sensibiliza os atores dos direitos humanos, abordando o conflito por meio da peça teatral, que estimula o pensamento divergente, e do polêmico diálogo que desenvolve o pensamento crítico em prol de novos conhecimentos. O método de pesquisa foi qualitativo, uma vez que trabalhou a partir de uma abordagem jurídica e em torno dos aspectos qualitativos da disciplina de direito. $\mathrm{O}$ exposto, com base na observação participante e não participante, um grupo focal, entrevistas e análise de conteúdo. Nesse caso, o universo escolhido foi um grupo limitado de professores de educação escolar. Para o registro das informações obtidas, foram utilizados instrumentos como formulários, entrevistas, palestras e prática pedagógica com a faixa etária. Em conclusão, trata-se de uma pesquisa interativa e participativa que fomentou a didática e rendeu dados relevantes para a sistematização da educação em direitos humanos por meio da pedagogia do teatro.

Palavras-chave: Peça teatral; Direitos humanos; Conflito e diálogo. 


\section{Introducción}

El presente artículo científico es un trabajo producto del estudio El sentido de la educación en derechos humanos a través de la pedagogía teatral: un campo de investigación en el aula, de Sandra Sofía Burmeister García, para el Magíster de Educación en Derechos Humanos (MEDH) realizado en el Centro de Cooperación Regional para la Educación de Adultos en América Latina y el Caribe (CREFAL, Pátzcuaro, México, 2017-2019) y la Secretaría de Educación Pública del Gobierno de México. En mérito de lo expuesto, se abordará uno de los objetivos específicos de la indagación global, formulado desde la siguiente pregunta: ¿Cómo generar una instancia de autoconocimiento y pensamiento crítico en la educación en derechos humanos a partir de una metodología activa?

La reflexión atenderá los conceptos señalados (autoconocimiento, educación en derechos humanos, pedagogía teatral, metodología activa y pensamiento crítico), mediante un cruce de saberes y en relación con la actualidad chilena. También, se compartirán algunos cuadros comparativos, aportes y conclusiones. Lo anterior se verá apoyado con un marco teórico sobre reflexiones de Fidel Sepúlveda, Verónica García-Huidobro, Isabel Rauber, entre otros investigadores. En virtud de un proceso académico y docente, cabe señalar que, durante la investigación primigenia, hubo salidas a terreno, entrevistas, reuniones diversas y clases prácticas con el grupo objetivo.

\section{Pedagogía teatral}

La pedagogía teatral es la disciplina que une el arte del teatro con el de la educación. Surgió en Europa después de la Segunda Guerra Mundial, dando respuesta a la necesidad de innovar en metodologías que fortalecieran el proceso de aprendizaje para la reconstrucción social, cultural, política y económica de aquel entonces. $\mathrm{Su}$ origen se remonta, prácticamente, al nacimiento de la Declaración Universal de los Derechos Humanos en 1948. Se basa en principios tales como: (1) ser una metodología activa, relativa al mundo afectivo de las personas; (2) priorizar el desarrollo de la vocación humana por sobre la artística; (3) entender la capacidad del juego dramático del ser humano como el recurso educativo fundamental; (4) respetar la naturaleza y las posibilidades objetivas de las personas, según la etapa del desarrollo del juego; (5) entender la herramienta como una actitud educativa más que como una técnica pedagógica, y (6) privilegiar el proceso de aprendizaje en lo artístico expresivo, más allá del resultado técnico teatral (García-Huidobro, 2008).

De preferencia, dicha disciplina se involucra en el desarrollo de las personas y ha tenido una evolución, en cuanto a tendencias, desde sus primeras manifestaciones. 
Por ejemplo, en la tendencia neoclásica caracterizada por la enseñanza de la técnica teatral pura, prima un resultado artístico y el sentido profesional del oficio. Luego, la tendencia progresista liberal, atiende el desarrollo afectivo de las personas y vuelca la emotividad a través de la expresión. Por otra parte, la tendencia radical instala la pedagogía teatral como un agente de cambio que transmite ideas y orienta aquellas decisiones que involucran al sistema social, religioso, político, cultural, ético y económico. En este caso, el pedagogo teatral es un facilitador de cambios sustanciales en las sociedades, al hacer uso del juego dramático o del teatro. La tendencia del socialismo crítico se vincula al trabajo docente, incorpora la noción del entorno y la diversidad, cumpliendo un rol social y crítico relacionado con la responsabilidad que se tiene por las personas (2008, p.19, 20).

En Chile, las áreas de inserción de la pedagogía teatral son 3. Primero, se introduce al interior del sistema educativo en la educación formal, apoyando los contenidos y objetivos básicos transversales de otros sectores curriculares, al ser la pedagogía teatral una herramienta pedagógica en el proceso de aprendizaje, ya fuere en la clase de lenguaje, matemáticas, idiomas, historia u otras asignaturas. En segundo lugar, la disciplina se incorpora como asignatura de expresión dramática (esto solo en algunos establecimientos educacionales y dependiendo de la visión educativa de la dirección), estimulando aptitudes expresivas del estudiantado. El foco es el desarrollo de las habilidades sociales. Para este caso, la pedagogía teatral considera 16 sesiones de expresión dramática que se abordan a partir de las etapas y subetapas del desarrollo del juego, entendiendo dichas fases como parte de las edades de 0 a 5 años, 5 a 9 años, 9 a 15 años y 15 a 25 años. La tercera forma de inserción es con el Programa de Estudio de Artes Escénicas (teatro y danza) para tercero o cuarto año de enseñanza media, que se instala en el área científico-humanista de la Reforma Educacional de 1990, con base en las definiciones del marco curricular de objetivos fundamentales y contenidos obligatorios de la Educación Media, definido en el Decreto 220, de mayo de 1998 (p. 21).

Por otro lado, la pedagogía teatral está presente al exterior del sistema educativo como educación no formal; así contribuye a las creaciones colectivas de un montaje teatral que propicie los códigos de comunicación, como taller de teatro vocacional. Asimismo, se configura en la dimensión terapéutica como taller de expresión artística, por lo que sirve de apoyo a la integración social (p. 22).

Adicional a lo anterior, la asignatura de educación teatral surgió desde un convenio de colaboración entre el Servicio Local de Educación Pública de Barrancas y la Fundación Teatro a Mil, en el 2016, junto con la directora del programa de Diplomados de la Escuela de Teatro UC, Verónica García-Huidobro, en el contexto del Nuevo Sistema de Educación Pública, creado dicho año mediante la Ley 21.040. 
Se estimó que, en el 2018 , más de 1000 estudiantes de 32 cursos de $5 .^{\circ}$ y $6 .^{\circ}$ básico incorporaron artes escénicas en su formación.

Como se puede ver, las áreas educativas, para aplicar la pedagogía teatral, son múltiples, tanto en la educación formal como en aquella no formal. En síntesis, cada una de las variedades de inserción abre una posibilidad para que el individuo se conecte consigo mismo y con otros, profundizando en las dimensiones del aprendizaje cognitivo, psicomotor y afectivo. De esta manera, la autoestima del sujeto participa activamente en un proceso pedagógico de autoconocimiento.

En el marco de la educación en derechos humanos, la pedagogía teatral - concebida en la investigación que aquí concierne - subyace desde la idea de fuerza que interpreta la educación mencionada, a través de una narrativa que hace avanzar esta pedagogía, con base en una perspectiva de derecho centrada en el sujeto. En este sentido, la pedagogía teatral es una herramienta de apoyo frente a las necesidades básicas de sectores menos privilegiados que se ven sometidos al factor del poder adquisitivo precario y a la falta de recursos económicos. Asimismo, al ser una disciplina flexible que da soporte a contenidos y asignaturas, es un instrumento indispensable para la educación en derechos humanos. Respecto a esta última, la realidad educativa del presente en el país demuestra que, en vez de progresar desde nuevos aprendizajes hacia una sociedad más democrática, todavía hay temas que no son abordados según las necesidades del Chile actual.

Dicho esto, se considera la educación basada en los derechos humanos como el cumplimiento de los elementos esenciales: acceso a la educación, el que esta sea de calidad y el respeto en el entorno del aprendizaje (Burmeister, 2017).

\section{Educación en derechos humanos}

En enero de 2021, en Chile, se rechazó, en la Cámara de Diputadas y Diputados, un proyecto de ley que proponía la creación de la asignatura denominada "Memoria y Derechos Humanos", para estudiantes de enseñanza básica y media. La iniciativa requería 89 respaldos para su aprobación, pero contó con 76 votos a favor, 48 en contra y 18 abstenciones. El proyecto proponía conocer y analizar las violaciones a los derechos humanos cometidas por la dictadura cívico-militar entre el 11 de septiembre de 1973 y el 11 de marzo de 1990.

Un sector político argumentó que los contenidos propuestos ya estarían abordados en otras asignaturas, por tanto, no sería necesaria la creación de una nueva. Según fundamentó el mismo sector político, esto significaría fomentar una historia incompleta de lo que vivió el país por esos años. El debate en la comisión de derechos 
humanos modificó la propuesta y estableció que este contenido se incorporaría en la ley que crea el Plan de Formación Ciudadana. Paso siguiente, el proyecto quedó archivado.

En alguna oportunidad, el Centro de Estudios y Promoción de los Derechos Humanos de la Universidad de la Frontera admitió, en su sitio web (año 2011), que en Chile la educación en derechos humanos ha sido promovida paulatinamente por el Estado, a partir de la década de los noventa; sin embargo, su incorporación plena está pendiente. También se consideró la encuesta realizada por el Instituto Nacional de Derechos Humanos, la cual arrojó que un 87, 2 \% de la población asocia los derechos humanos a crímenes cometidos durante la dictadura. Otros derechos, a la vivienda y salud, rodearon el 55,9\%. Por último, el derecho a la no discriminación de minorías representó a un 30,7\%.

Ante lo dicho, defender y proteger los derechos humanos depende, en gran medida, del conocimiento que la población tenga sobre ellos. Así, la educación (formal y no formal) cumple un rol fundamental como facilitadora. Entonces, es posible comprender la educación en derechos humanos como una necesidad que debe estar presente en la educación formal escolar y universitaria, al igual que, en lo posible, en la no formal. Con ella, se podría elaborar una pedagogía ética, crítica y política que se comprometiese a construir modos de vida más justos, mediante la práctica de derechos enfocados en valores inherentes a una cultura de respeto.

Una mirada comprensiva frente a la educación en derechos humanos se entiende como un privilegio que promueve el respeto universal y efectivo de todos los derechos humanos y las libertades fundamentales. De esta manera, se empodera a las personas desde una postura responsable, conforme a su realidad histórica. Asimismo, se atiende a las violaciones de derechos humanos y a las víctimas de abusos, para que puedan reconocerse como sujetos de derecho en la sociedad.

El Estado es el principal garante de los derechos humanos y el responsable, ante la sociedad, de prevenir, investigar, sancionar y reparar aquellas violaciones a ellos. Esos derechos están presentes para regular a las autoridades y servir a la ciudadanía. Entre los asuntos que se deben aprender, está que no todo delito es una violación a los derechos humanos, sino solo aquellos crímenes cometidos por agentes del Estado en contra de un ciudadano o de varios.

Desde la formación en derechos humanos, se desarrollan habilidades y actitudes que promueven igualdad, dignidad y respeto en la comunidad, para conocer mejor 
el entorno. Por tanto, dicha formación es para toda persona y el aprendizaje de aquellos se puede adquirir en cualquier edad y durante toda la vida.

En el libro La Escuela: como territorio de intervención política, Isabel Rauber señala, en su ensayo sobre educadores y políticos, que educar implica un reconocimiento del saber de los demás, y que el eje central de la batalla está en lo cultural. Asimismo, para que las cosas funcionen en materia educativa, se debe construir cimientos sólidos y no improvisar en soluciones inmediatas. La transformación está en la persona individual y en la organización. La autora menciona las dimensiones para analizar la realidad y se refiere a una quinta, explicando que surge al momento en que las personas se consideran sujetos activos desde su capacidad, al cambiar algo; dimensión que debe ser creada por el sujeto que potencia y sintetiza una acción transformadora. En otras palabras, esto sería imaginar la nueva orgánica, el nuevo barrio, la nueva familia, la nueva ciudad o el país renovado. En ello radica la nueva escuela y el sentido que tiene el educador, como un constructor o arquitecto, capaz de inventar y hacer realidad lo que ha ingeniado. La realidad es solo el punto de partida, inclusive si fuere desde un diagnóstico que ha concluido en la imposibilidad de cambiarla. Crear la quinta dimensión es una labor colectiva que solo puede existir teniendo los pies en la tierra. La construcción de un movimiento político-social es abriendo una puerta al crecimiento territorial de algo concreto que tiene que ver con el crear e inventar. Enfatiza que la conciencia, la escuela y la cultura van de la mano. La comunidad educativa tiene la capacidad de ubicar a la escuela como un actor sociopolítico activo y vivo de una comunidad y entre comunidades (Zemelman, Rauber y Tamarit, 2004, pp. 101-115).

De lo anterior, se podría decir que cuando la educación en derechos humanos analiza el proceso de aprendizaje se vuelve crítica y sensibiliza. Al ser explicada a través de la pedagogía teatral, facilita al sujeto interiorizarse en un estado reflexivo lúdico. En este sentido, el pedagogo Paulo Freire menciona que el sujeto abre su mente ante el obstáculo, para trascenderlo desde la intención de la conciencia, mediante la formulación, a interrogantes, en lo que se refiere a una educación emancipadora. Así se descubre a sí mismo (2009, pp. 17-18).

En este sentido, se puede hacer un paralelo entre la pedagogía teatral, la educación en derechos humanos y la pedagogía crítica, ya que, al ser cualitativas, antiautoritarias y libertadoras, plantean un desarrollo individual en el colectivo, con la creencia en la nueva relación dialógica entre profesor y estudiante, siendo la palabra la portadora de la dignidad a través de la praxis y del diálogo transversal. Por ejemplo: la pedagogía crítica examinará cómo la estructura educativa y el currículo dan forma al conocimiento. Mientras, la educación en derechos humanos indagará cómo se moldea al sujeto de derecho, lo empoderará para analizar la interrelación 
entre educación y sociedad. A su vez, la pedagogía teatral se encargará del pleno desarrollo del sujeto, por medio de una metodología activa de autoconocimiento individual y grupal dentro o fuera del aula.

Al traer al presente las reflexiones del maestro Fidel Sepúlveda, en cuanto a la misión de educar del profesor, se vislumbra que esta no viene desde el currículo profesional, sino que es algo propio de la ciencia del saber vivir, de un arte: el arte de vivir. Educar es una operación mayéutica por la que el hombre da a luz su humanidad. Este diálogo metódico de la filosofía socrática en que el interlocutor interpelado descubre las verdades por sí mismo. En esa línea, la palabra poética es el monumento vivo que encarna la historia. La creatividad del sujeto subyace ante su expresividad, al hacer viva su voz desde su propio conocimiento. Y es mediante esto que se va fundando la identidad de ese sujeto, como algo revelador. Entonces, un plan educativo debe estar orientado a recuperar la memoria y la imaginación del pueblo chileno, ya que la educación pasa por mirarse hacia adentro (2015, pp. 147-155).

\section{Metodología}

En la investigación global, es importante señalar que la pedagogía teatral ha sido instalada en el aula como una didáctica especial en el campo cooperativo de la acción docente. Es entendida como una metodología activa en el aula, la cual se enfoca en el proceso de aprendizaje, incorporando el juego en el aspecto psicológico, motriz, expresivo, pedagógico y social. Sumado a ello, surgen algunos conceptos lúdicos adheridos a las investigaciones de Jean Piaget, Lev Vygotski y Karl Groos. La clase en sí misma se conoce como Sesión de Expresión Dramática y se divide en 5 fases: preliminares, sensibilización, creatividad vocal, creatividad corporal y expresión.

Cada sesión de expresión dramática será una experiencia distinta, porque se trabaja con seres humanos en su diversidad. Cada persona aporta vivencias de su propio contexto social, histórico, anímico, educativo, familiar, religioso, entre otros. Según eso, se enfrentará al conflicto con un punto de vista. La intervención pedagógica aborda emociones, sentimientos y pensamientos tanto individuales como colectivos, lo que muchas veces puede parecer complejo, en virtud, de conciliar diferencias.

De esta manera, se aborda el juego teatral con un rol específico frente al conflicto, comprendido el segundo como la controversia que tiende a surgir durante el diálogo y que se observa en la dramatización e improvisación, mientras ocurre la experiencia pedagógica en el aula. 
En la Sesión de Expresión Dramática, se contempla práctica, teoría y nexo pedagógico; con ello, además, la valoración (evaluación) perceptual y conceptual, individual y grupal. Para efectos de pautas de evaluación o descriptores, las dimensiones que se consideran son cualitativas, por ejemplo: creatividad, participación, voz, trabajo en equipo, expresión, entre otras. Los niveles también son cualitativos y ligados a una nota final, por ejemplo: muchísimo (7.0) / bastante (6.0) / moderado (5.0) / regular (4.0) / poco (3.0) / nada (2.0) y no se presenta (1.0) 2 .

\section{Resultados y análisis de resultados}

La formación en derechos humanos a través de la pedagogía teatral funciona como un faro lúdico y sistemático, para superar la educación estandarizada y avanzar hacia planteamientos educativos integrales.

El diagnóstico de la investigación tuvo un impacto inicial con un alcance de 36 profesores de educación formal, con cursos de 35 estudiantes, y se extendió informalmente a las familias. Se podría hablar de más de 1200 alumnos, aproximadamente.

El universo de estudio arrojó un gráfico con un $83 \%$ de los participantes que no sabía sobre derechos humanos. Hubo un $8 \%$ que sabía algo. Un $3 \%$ sabía y un $2,5 \%$ no respondió.

2 En Chile, las calificaciones educativas "llamadas coloquialmente notas" tienen una escala única numérica, del 1,0 al 7,0 con máximo un decimal, utilizada tanto en la educación primaria y secundaria como en la superior. 


\section{Profesores}

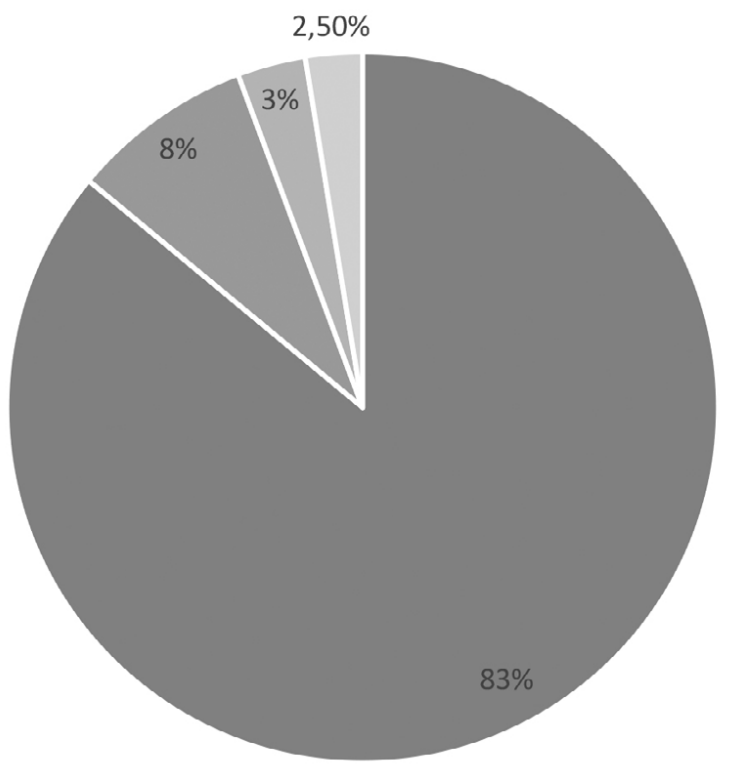

Gráfico 1. Universo de estudio (36 profesores de educación escolar) para la investigación preliminar realizada en el Módulo de Derechos Humanos de la PUC. (Burmeister, 2019)

El gráfico muestra una aproximación (al detalle cuantificable) al problema investigativo, que era la desconexión de los profesores, en referencia a su formación en derechos humanos; surgió, de tal modo, el cuestionamiento sobre cómo sensibilizar a los docentes en derechos humanos. Dicho sea de paso, esto se realizó con el fin de intervenir la comunidad escolar, en vías de una convivencia más pacífica.

La investigación, sobre educación en derechos humanos a través de la pedagogía teatral, reunió saberes; hizo de la clase activa el contexto ideal para la práctica de una pedagogía crítica, liberadora y responsable del otro. Por tanto, esta contribuyó a fomentar la transformación social en términos de emancipación, democracia, igualdad, ciudadanía, justicia e inclusión.

Ejemplos sobre esto se exponen a continuación.

Sobre la emancipación: Se manifestó, a través de una educación sistémica efectiva, que el desarrollo humano entre estudiante y maestro fue vinculado a la comunidad. Por otra parte, se vio reflejada la autonomía de los alumnos y su libertad de 
pensamiento. Se abordó la reflexión crítica y divergente, desde argumentos y experiencias que denotaron empoderamiento. Se manifestaron relaciones dialógicas durante el juego teatral y el diálogo controversial sin consenso. Se pudo sostener una comunicación sincera, en vías de una convivencia pacífica.

Sobre democracia: Los estudiantes mostraron una actitud más activa, a partir de un aprendizaje mutuo, junto al maestro y frente al trabajo en equipo. La práctica del docente como mediador de conocimientos se consolidó mediante el proceso de aprendizaje. El profesor no practicó autoritarismo, sino una comunicación grupal productiva. Lo anterior fue generando una participación en comunidad desde una dimensión política. Hubo diversidad de opinión y libertad expresiva en el aula.

Sobre igualdad: De preferencia, se ensayó la formación de sujetos de derecho con una estrategia transversal controversial educativa. En cuanto a la justicia social, se comprendió cómo funciona la redistribución del ingreso, al igual que cómo afecta el rendimiento escolar y a la violencia escolar. Con esto se pudo observar aquellas tendencias más cercanas a las mismas oportunidades y los derechos. Se valoró el derecho de expresión por parte del grupo.

Sobre ciudadanía: La identidad hacia los derechos humanos en la vida cotidiana fue una instancia fija de considerar. Se mantuvo la práctica reflexiva en la formación axiológica de la realidad en la educación escolar. La promoción formativa en derechos humanos fue vista desde una dimensión sensitiva, respecto a la vida cotidiana y al contexto social e histórico. Se pudo reevaluar la educación cívica como una instancia formadora esencial. El trabajo comunitario se valoró como una oportunidad de desarrollo humano en redes populares que propician al crecimiento de una comuna.

Sobre justicia social: Se entendió que la injusticia socioeconómica tiene que ver con la explotación y marginación de las personas, al privarlas de bienes materiales. Se comprendió la injusticia cultural o simbólica frente a patrones sociales, como los valores diversos entre las personas. Hubo un hallazgo sobre el problema de coherencia entre la interpretación y comunicación, que repercute en la autorrealización del individuo. Por otra parte, la injusticia de lucha de identidades se observó desde una dimensión política, económica y cultural. Se valoró la educación y se evaluó con un enfoque de derecho. Se abordó la comprensión de la equidad como reconocimiento de las diferencias.

Sobre inclusión: Se presentaron las tendencias pedagógicas incluyentes, por ejemplo, la pedagogía del oprimido de Paulo Freire y su visión pedagógica, con un lenguaje genuino entre maestro y estudiante. También, se trabajó con la 
comprensión de una pedagogía progresista y libertadora, donde el cambio social y el multiculturalismo advierten un carácter sociopolítico. Se absorbió el significado de la pedagogía teatral, su rol educativo, social y terapéutico. Hubo un análisis sobre lo trascendente del juego y la experiencia creativa en el aula para el desarrollo del sujeto: percepción, sensaciones, imaginación, improvisación. Se abordó la importancia de la atención a los indicadores de derecho en la educación (Tomasevski, s. f.).

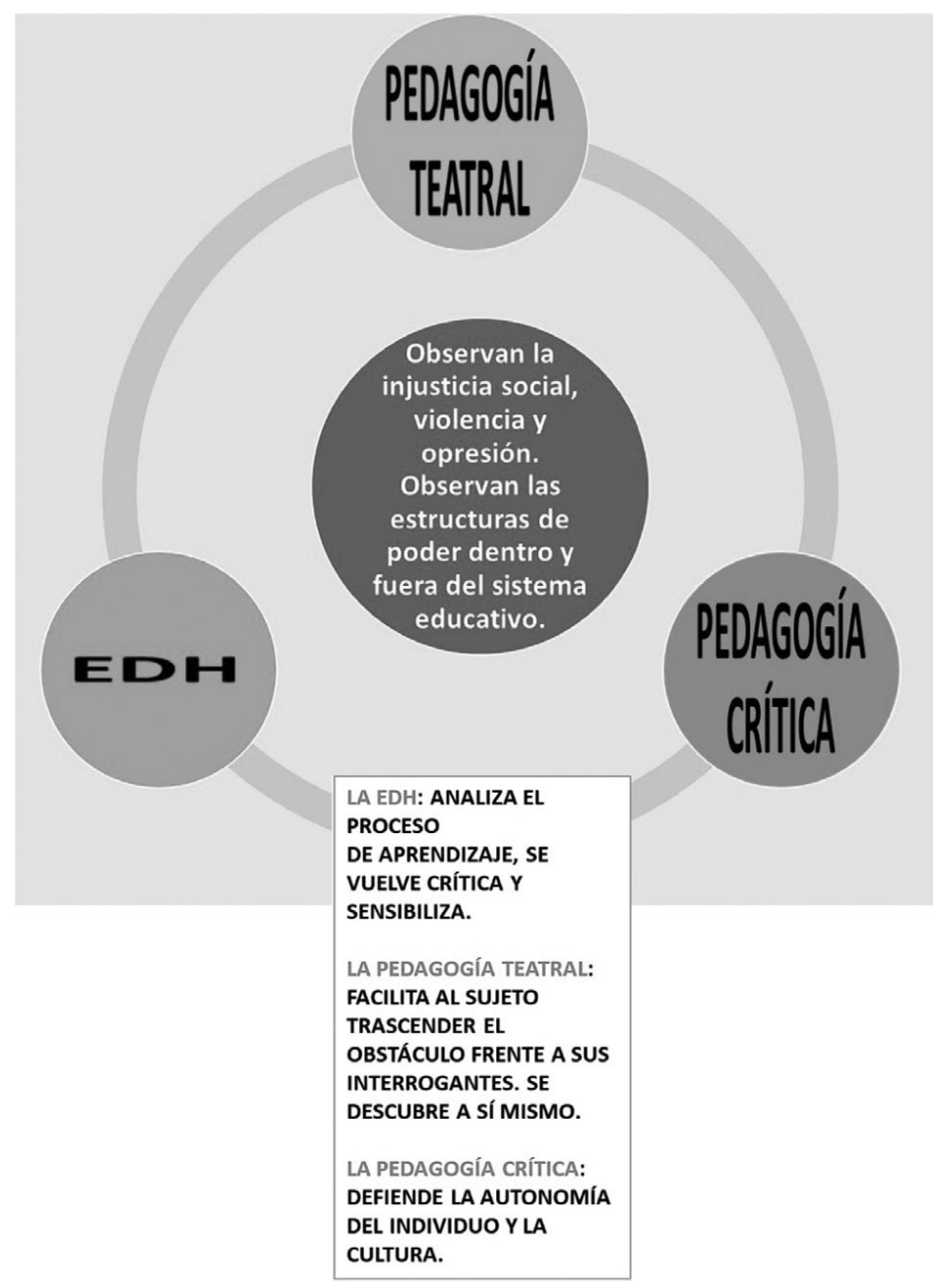

Cuadro 1. Comparativa entre pedagogía teatrla, pedagogía crítica y educación en derechos humanos. Contenidos del proceso de sistematización y contribución de la tesis: "El sentido de la educación en derechos humanos a través de la pedagogía teatral: un campo de investigación en el aula". (Burmeister, 2019) 
Hubo un plan de transferencia tentativo para realizar, entre los años 2018 y 2019, pero se vio frustrado por falta de seguimiento que ofreciera una comparación paralela de contenidos y aprendizajes de cada establecimiento educacional.

Sí se pudo llevar a cabo una triangulación cualitativa importante entre las Bases de Educación en Derechos Humanos, los Principios de la Pedagogía Teatral y las Orientaciones de los Objetivos Fundamentales Transversales.

La muestra ofreció parámetros comparativos sobre afectividad, autoestima, humanización del aprendizaje, expresividad del sujeto, autonomía del sujeto, justicia social en el aula y equidad en el aprendizaje, que se pueden observar seguidamente:

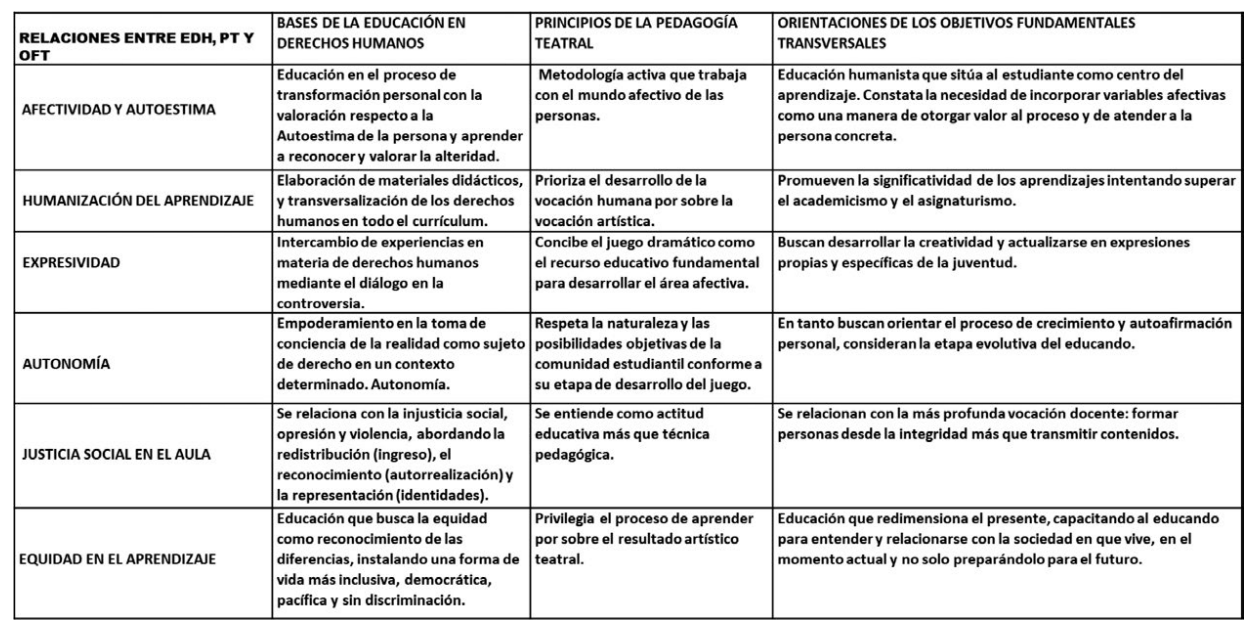

Cuadro 2. "Relación entre EDH, PT y OFT".

Fuente: Fraser, N. y Honneth , A. (2006). García-Hiuidobro, V. (2008). Magendzo, A. y Pavez, J. (2015). Ponce, L. (2011). Reimers, F. (2000) y López, M. (2004).

\section{Conclusiones}

El sentido de la educación en derechos humanos a través de la pedagogía teatral permite avanzar desde una perspectiva de derecho, fortaleciendo el objeto de la formación y considerando la calidad de vida en el aula.

La pedagogía teatral centra su energía en la persona humana. Algo semejante a la comunión entre maestro y estudiante. No solo el pensamiento crítico será lo que revele condicionamientos y libertades, sino también los afectos de las personas según su contexto. 
La educación en derechos humanos a través de la pedagogía teatral contribuye a un ejercicio constante de autoconocimiento, a partir de una metodología activa, práctica y participativa, que estimula el pensamiento crítico tanto en el desarrollo individual como en el trabajo colectivo.

La educación en derechos humanos, la pedagogía teatral y los objetivos fundamentales transversales son la vanguardia en la práctica docente, en función de una educación más efectiva y afectiva, potenciando la autonomía del sujeto.

La formación educativa en derechos humanos a través de la pedagogía teatral ofrece herramientas, superando la educación estandarizada y avanzando hacia planteamientos formativos integrales que fortalecen el desarrollo del pensamiento crítico.

La educación en derechos humanos, la pedagogía teatral y la pedagogía crítica permiten generar nuevos conocimientos, compartir saberes y crear conciencia de derecho, a través del diálogo controversial que fortalece la reflexión respecto del entorno. Asimismo, tales disciplinas abordan una relación directa con la injusticia social, la opresión y la violencia. Todas ellas observan las estructuras de poder dentro y fuera del sistema educativo.

Es evidente, entonces, que la educación en derechos humanos, la pedagogía teatral y la pedagogía crítica amplían la atención hacia el valor de lo cualitativo, al captar las experiencias formativas, en vías de un desarrollo integral de la personalidad del sujeto, tanto en la horizontalidad de relaciones (de Zemelman, Rauber y Tamarit, 2004) y roles como en la controversia de ideas y en la corrección de prácticas discriminatorias.

Finalmente, la educación en derechos humanos por medio de la pedagogía teatral es un modelo de sistematización educativa, el cual permite profundizar cualitativamente en el aula y alude al deseo de una filosofía sana que reúne cultura, arte y ciencia. 


\section{Referencias}

Burmeister, S. (2017). "Derecho a la educación en Chile". https://www.academia. edu/35548021/Derecho_a_la_Educación_en_Chile

Centro de Estudios y Promoción de los Derechos Humanos (2011). Educación en derechos humanos en Chile. Universidad de la Frontera. http://derechoshumanos.ufro.cl/index. $\mathrm{php} /$ login/213-educacion-en-derechos-humanos-en-chile

Freire, P. (2009). Pedagogía del oprimido (Mellado, J., ed. y trad.). 3. a ed., 1. a reimp., Buenos Aires. Siglo Veintiuno Editores Argentina; Tierra Nueva. (Original publicado en 1970).

García-Huidobro, V. (2008). Pedagogía Teatral: metodología activa en el aula. Ediciones Universidad Católica de Chile; Salesianos Impresores S. A. (Original publicado en 2004).

Sepúlveda, F. (2015). Arte vida. 1. ${ }^{\text {a }}$ ed, Santiago de Chile. Liberalia Ediciones Ltda.

Tomasevski, K. (s. f.). Indicadores del Derecho a la Educación. http://www.derechoshumanos.unlp.edu.ar/assets/files/documentos/indicadores-del-derecho-a-la-educacion.pdf

Zemelman, H., Rauber, I., y Tamarit, J. (2004). La escuela: como territorio de intervención política. 1. ${ }^{a}$ ed. Confederación de Trabajadores de la Educación de la República de Argentina. Secretaría de Educación. Capital Federal. 
